\title{
Basic properties of an alternative flow equation in gravity theories
}

\author{
Peter M. Lavrov $(a, b)[1]$ \\ (a) Tomsk State Pedagogical University, \\ Kievskaya St. 60, 634061 Tomsk, Russia \\ (b) National Research Tomsk State University, \\ Lenin Av. 36, 634050 Tomsk, Russia
}

\begin{abstract}
Basic properties of alternative flow equation in Quantum Gravity are studied. It is shown that the alternative flow equation for effective two-particle irreducible effective action is gauge independent and does not depend on IR parameter $k$ on shell.
\end{abstract}

Keywords: Gauge dependence, functional renormalization group, effective two-particle irreducible action, Quantum Gravity

PACS numbers: 11.10.Ef, 11.15.Bt

\footnotetext{
${ }^{1}$ E-mail: lavrov@tspu.edu.ru
} 


\section{Introduction}

Being popular among the functional renormalization group (FRG) community the standard formulation of the FRG approach 1, 2] (among recent reviews of the method see, for example, [3]) meets with the very serious gauge dependence problem of the effective average action as within the perturbation theory [4] as well as on the level when it is found as a non-perturbative solution to the flow equation making impossible physical interpretations of any results obtained in gauge theories with the help of effective average action [5, 6].

Recently it has been proposed [7] and studied [8] alternative methods in comparison with [4] when the regulators being essential tools of the FRG are considered as sources to composite operators. It differs with the standard introduction of external sources to composite operators [9] (generalizations to the case of gauge theories have been proposed in [10, 11, 12]) when they are usual functions of space-time coordinates instead of the regulators which are some differential operators in general [13]. In contrast with the standard FRG [1, 2] the alternative methods [7, 8] lead to the 2PI effective average actions which have good properties within the perturbation theory. Unfortunately they still unacceptable on the non-perturbative level because solutions to corresponding flow equations depend on gauge at any value of the IR parameter $k$ including the fixed points [8].

In the present paper we are going to extend the reformulation of the FRG proposed in [4 for Yang-Mills fields to the case of gravity theories and to study basic properties of corresponding flow equation. The paper is organized as follows. In Sec. 2 the properties of effective action on an arbitrary background metric for gravity theories in the standard quantization scheme [16] are presented. In Sec. 3 the gauge dependence problem of 2PI irreducible effective average action for Quantum Gravity within the perturbation theory is discussed. In Sec. 4 the derivation of alternative flow equation for the effective action with composite operators and the study its $k$-dependence are given. In Sec. 5 the gauge dependence of the alternative flow equations is investigated. Finally, in Sec. 6 the results obtained in the paper are discussed.

The DeWitt's condensed notations [14] are used. The functional derivatives with respect to fields and sources are considered as right and left correspondingly. The left functional derivatives with respect to fields are marked by special symbol " $\rightarrow$ ". Arguments of any functional are enclosed in square brackets [ ], and arguments of any function are enclosed in parentheses, ( ). The symbol $F_{, i}[\phi, \ldots]$ means the right derivative of $F[\phi, \ldots]$ with respect to field $\phi^{i}$. 


\section{Effective action for gravity theories}

We start with an arbitrary initial action $S_{0}[g]$ of the metric tensor $g=\left\{g_{\mu \nu}\right\} 2$ We suppose the invariance of $S_{0}[g]$,

$$
\delta_{\xi} S_{0}[g]=0
$$

under general coordinate transformations which infinitesimally take the form of gauge transformations of $g_{\mu \nu}$

$$
\delta_{\xi} g_{\mu \nu}=-\partial_{\sigma} g_{\mu \nu} \xi^{\sigma}-g_{\mu \sigma} \partial_{\nu} \xi^{\sigma}-g_{\sigma \nu} \partial_{\mu} \xi^{\sigma}=R_{\mu \nu \sigma}(g) \xi^{\sigma}
$$

where $\xi^{\sigma}$ are arbitrary functions of space-time coordinates and $R_{\mu \nu \sigma}(g)$ are the gauge generators satisfying the closed and irreducible gauge algebra (for detailed description see [15]),

$$
\left[\delta_{\xi_{1}}, \delta_{\xi_{2}}\right] g_{\mu \nu}=\delta_{\xi_{3}} g_{\mu \nu}, \quad \xi_{3}^{\sigma}=F_{\alpha \beta}^{\sigma} \xi_{2}^{\beta} \xi_{1}^{\alpha}=\xi_{1}^{\sigma} \partial_{\alpha} \xi_{2}^{\alpha}-\xi_{2}^{\sigma} \partial_{\alpha} \xi_{1}^{\alpha}
$$

In (2.3) $F_{\alpha \beta}^{\sigma}$ are structure coefficients of gauge algebra which do not depend on fields $g_{\mu \nu}$ and have the universal form for any gravity theory.

On quantum level one operates with the action

$$
S[\phi, \bar{g}]=S_{0}[h+\bar{g}]+S_{g h}[\phi, \bar{g}]+S_{g f}[\phi, \bar{g}],
$$

appearing in the Faddeev-Popov method [16]. Here the decomposition of $g, g=h+\bar{g}$, on a background metric $\bar{g}=\left\{\bar{g}_{\mu \nu}\right\}$ and quantum fluctuation $h=\left\{h_{\mu \nu}\right\}$ is used. In (2.4) $\phi^{i}=$ $\left(h_{\alpha \beta}, B^{\alpha}, C^{\alpha}, \bar{C}^{\alpha}\right)$ is the set of quantum fields, $C^{\alpha}, \bar{C}^{\alpha}$ are the ghost and antighost fields, $B^{\alpha}$ are auxiliary Nakanishi-Lautrup fields for introducing the gauge fixing functions, $\chi_{\alpha}(\bar{g}, h)$. A standard choice of $\chi_{\alpha}(\bar{g}, h)$ corresponding to the background field gauge condition in linear gauges [17] reads

$$
\chi_{\alpha}(\bar{g}, h)=\mathcal{F}_{\alpha}^{\mu \nu}(\bar{g}) h_{\mu \nu}, \quad \mathcal{F}_{\alpha}^{\mu \nu}(\bar{g})=-\bar{g}^{\mu \sigma}\left(a \delta_{\alpha}^{\nu} \bar{\nabla}_{\sigma}+b \delta_{\sigma}^{\nu} \bar{\nabla}_{\alpha}\right)
$$

where $\bar{\nabla}_{\sigma}$ is the covariant derivative constructed with the help of $\bar{g}_{\mu \nu}$ and $a, b$ are constants. The de Donder gauge condition corresponds to the case when $a=1, b=1 / 2 . S_{g h}[\phi, \bar{g}]$ is the ghost action,

$$
S_{g h}[\phi, \bar{g}]=\int d x \sqrt{-\operatorname{det} \bar{g}} \bar{C}^{\alpha} G_{\alpha}^{\beta \gamma}(\bar{g}, h) R_{\beta \gamma \sigma}(\bar{g}+h) C^{\sigma},
$$

with the notation

$$
G_{\alpha}^{\beta \gamma}(\bar{g}, h)=\frac{\delta \chi_{\alpha}(\bar{g}, h)}{\delta h_{\beta \gamma}}
$$

\footnotetext{
${ }^{2}$ Standard examples are Einstein gravity, $S_{0}(g)=\kappa^{-2} \int d x \sqrt{-\operatorname{det} g} R$, and $R^{2}$ gravity, $S_{0}(g)=$ $\int d x \sqrt{-\operatorname{det} g}\left(\lambda_{1} R^{2}+\lambda_{2} R^{\mu \nu} R_{\mu \nu}+\kappa^{-2} R\right)$.
} 
and $S_{g f}[\phi, \bar{g}]$ is the gauge fixing action

$$
S_{g f}[\phi, \bar{g}]=\int d x \sqrt{-\operatorname{det} \bar{g}} B^{\alpha} \chi_{\alpha}(\bar{g}, h) .
$$

For any admissible choice of gauge fixing functions $\chi_{\alpha}(\bar{g}, h)$ the action (2.4) is invariant under global supersymmetry (BRST symmetry) [18, 19], 3

$$
\delta_{B} h_{\mu \nu}=R_{\mu \nu \alpha}(\bar{g}+h) C^{\alpha} \Lambda, \quad \delta_{B} B^{\alpha}=0, \quad \delta_{B} C^{\alpha}=-C^{\sigma} \partial_{\sigma} C^{\alpha} \Lambda, \quad \delta_{B} \bar{C}^{\alpha}=B^{\alpha} \Lambda,
$$

where $\Lambda$ is a constant Grassmann parameter.

The generating functional of Green functions, $Z=Z[J, \bar{g}]$, is constructed in the form of functional integral

$$
Z[J, \bar{g}]=\int D \phi \exp \left\{\frac{i}{\hbar}(S[\phi, \bar{g}]+J \phi)\right\}=\exp \left\{\frac{i}{\hbar} W[J, \bar{g}]\right\},
$$

where $W=W[J, \bar{g}]$ is the generating functional of connected Green functions and $J=\left\{J_{i}\right\}$ is the set of external sources to fields $\phi=\left\{\phi^{i}\right\}$. The generating functional of vertex functions (effective action), $\Gamma=\Gamma[\Phi, \bar{g}]$, is defined through the Legendre transform of $W$,

$$
\Gamma[\Phi, \bar{g}]=W[J, \bar{g}]-J \Phi, \quad \Phi^{i}=\frac{\delta W}{\delta J_{i}}, \quad \frac{\delta \Gamma}{\delta \Phi^{i}}=-J_{i}
$$

and can be found as a solution to the following functional integro-differential equation,

$$
\exp \left\{\frac{i}{\hbar} \Gamma[\Phi, \bar{g}]\right\}=\int D \phi \exp \left\{\frac{i}{\hbar}\left(S[\Phi+\phi, \bar{g}]-\frac{\delta \Gamma[\Phi, \bar{g}]}{\delta \Phi} \phi\right)\right\}
$$

The standard approach (perturbation theory) in quantum field theory to find a solution to the Eq. (2.12) is based on using loop expansions

$$
\Gamma[\Phi, \bar{g}]=S[\Phi, \bar{g}]+\hbar \Gamma_{1}[\Phi, \bar{g}]+\ldots
$$

where

$$
\Gamma_{1}[\Phi, \bar{g}]=-i \operatorname{Tr} \ln \left(S^{(2)}[\Phi, \bar{g}]\right), \quad S^{(2)}[\Phi, \bar{g}]=\frac{\delta^{2} S[\Phi, \bar{g}]}{\delta \Phi \delta \Phi}
$$

is one-loop approximation and ellipses mean higher order loop contributions.

The generating functionals $Z, W, \Gamma$ depend on gauges but due to the BRST symmetry the gauge dependence has a very special form and for variation $\delta \Gamma$ under an infinitesimal change of gauge fixing functions, $\chi \rightarrow \chi+\delta \chi$, obeys the property

$$
\left.\delta_{\chi} \Gamma\right|_{\frac{\delta \Gamma}{\delta \Phi}=0}=0
$$

\footnotetext{
${ }^{3}$ The gravitational BRST transformations were introduced in [20, 21, 22, For more compact presentation of the BRST transformations we use the notation $\delta_{B}$ for $\delta_{B R S T}$.
} 
i.e. it does not depend on gauges when it is considered on extremals. For the first time the gauge dependence of effective action for gravity theories in the form (2.15) has been described in [23] (for more early descriptions of gauge dependence of effective action in gauge theories see papers [24, 25, 26, 27, 28]). This fact allows to state the gauge independence of $S$-matrix thanks to the equivalence theorem [29]. Among other important properties being very useful in practical calculations within the background field method [30, 31, 32] the functionals

$$
Z[\bar{g}]=Z[J=0, \bar{g}], \quad W[\bar{g}]=W[J=0, \bar{g}], \quad \Gamma[\bar{g}]=\Gamma[\Phi=0, \bar{g}]
$$

are covariant functionals with respect to $\bar{g}$,

$$
\delta_{\xi} Z[\bar{g}]=0, \quad \delta_{\xi} W[\bar{g}]=0, \quad \delta_{\xi} \Gamma[\bar{g}]=0,
$$

as well as they do not depend on gauges

$$
\delta_{\chi} Z[\bar{g}]=0, \quad \delta_{\chi} W[\bar{g}]=0, \quad \delta_{\chi} \Gamma[\bar{g}]=0,
$$

as the direct consequences of the BRST invariance of action $S[\phi, \bar{g}][33$.

\section{Gauge dependence of modified effective average action}

The effective average action of the FRG [1, 2] is ill-defined perturbatively in the case of YangMills theories [4, 6] and gravity theories [15] because of the gauge dependence of effective average action on-shell. To improve the situation for Quantum Gravity we apply the background field method [30, 31, 32] 4 and the formulation of effective action with composite operators [9, 10, 11] for construction of modified effective average action in the form used for the first time in the case of Yang-Mills theories in [4].

With the help of addition of a scale-dependent regulator action, $S_{k}$, being quadratic in the quantum fields, the FRG modifies behavior of propagators of quantum fields in IR and UV regions [1, 2]. In the case of Quantum Gravity the scale-dependent regulator action takes the form [13]

$$
\begin{aligned}
S_{k}(\phi, \bar{g}) & =\int d x \sqrt{-\operatorname{det} \bar{g}}\left[\frac{1}{2} h_{\mu \nu} R_{k}^{(1) \mu \nu \alpha \beta}(\bar{g}) h_{\alpha \beta}+\bar{C}^{\alpha} R_{k \alpha \beta}^{(2)}(\bar{g}) C^{\beta}\right] \equiv \\
& \equiv \int d x \sqrt{-\operatorname{det} \bar{g}}\left(\mathcal{L}_{k}^{(1)}(h, \bar{g})+\mathcal{L}_{k}^{(2)}(C, \bar{C}, \bar{g})\right)
\end{aligned}
$$

where $R_{k}^{(1) \mu \nu \alpha \beta}(\bar{g}), R_{k \alpha \beta}^{(2)}(\bar{g})$ are regulators with properties

$$
R_{k}^{(1) \mu \nu \alpha \beta}(\bar{g})=R_{k}^{(1) \alpha \beta \mu \nu}(\bar{g}), \quad \lim _{k \rightarrow 0} R_{k}^{(1) \mu \nu \alpha \beta}(\bar{g})=0, \quad \lim _{k \rightarrow 0} R_{k \alpha \beta}^{(2)}(\bar{g})=0 .
$$

On quantum level the FRG operates with the full action

$$
S_{W k}[\phi, \bar{g}]=S[\phi, \bar{g}]+S_{k}[\phi, \bar{g}]
$$

\footnotetext{
${ }^{4}$ For recent development of the background field method see [34].
} 
where $S[\phi, \bar{g}]$ is defined in (2.4) - (2.8). The action $S_{W k}[\phi, \bar{g}](\underline{3.3})$ is not invariant under the BRST transformations (2.9) that leads to the gauge dependence problem within the FRG for Quantum Gravity [15].

As it was already mentioned above to improve the situation we propose following to [4] the generating functional of Green functions $Z_{k}=Z_{k}[J, \bar{g}, \Sigma]$ in the form

$$
Z_{k}=\int D \phi \exp \left\{\frac{i}{\hbar}\left(S[\phi, \bar{g}]+J \phi+\Sigma \mathcal{L}_{k}(\phi, \bar{g})\right)\right\}
$$

where $\Sigma=\left(\Sigma_{1}, \Sigma_{2}\right)$ are external sources to composite fields $\mathcal{L}_{k}(\phi, \bar{g})=\left(\mathcal{L}_{k}^{(1)}(h, \bar{g}), \mathcal{L}_{k}^{(2)}(C, \bar{C}, \bar{g})\right)$ and $J=\left\{J_{i}\right\}$ are external sources to fields $\phi=\left\{\phi^{i}\right\}$. Let us note that from the definition of generating functional $Z_{k}$ (3.4) and properties of the regulators (3.2) it immediately follows coincidence of the functional $Z_{k}$ with the standard generating functional of Green functions in the limit $k \rightarrow 0$. The same statement is valid for the corresponding S-matrices.

Now we are going to study dependence of the functional $Z_{k}[J, \bar{g}, \Sigma](3.4)$ on gauges. To simplify the corresponding presentation it is useful to rewrite the action $S[\phi, \bar{g}]$ in the form

$$
S[\phi, \bar{g}]=S_{0}[h+\bar{g}]+\Psi[\phi, \bar{g}] \hat{R}[\phi, \bar{g}]
$$

with the help of gauge fixing functional $\Psi[\phi, \bar{g}]$

$$
\Psi[\phi, \bar{g}]=\int d x \sqrt{-\operatorname{det} \bar{g}} \bar{C}^{\alpha} \chi_{\alpha}(\bar{g}, h),
$$

containing all information about gauge fixing, and with the generator of BRST transformations (2.9)

$$
\hat{R}[\phi, \bar{g}]=\int d x \frac{\overleftarrow{\delta}}{\delta \phi^{i}} R^{i}(\phi, \bar{g}), \quad R^{i}(\phi, \bar{g})=\left(R_{\mu \nu \sigma}(\bar{g}+h) C^{\sigma}, 0,-C^{\sigma} \partial_{\sigma} C^{\alpha}, B^{\alpha}\right)
$$

Consider an infinitesimal variation of gauge fixing functions, $\chi_{\alpha}(\bar{g}, h) \rightarrow \chi_{\alpha}(\bar{g}, h)+\delta \chi_{\alpha}(\bar{g}, h)$ which causes the variation of gauge fixing functional, $\Psi[\phi, \bar{g}] \rightarrow \Psi[\phi, \bar{g}]+\delta \Psi[\phi, \bar{g}]$. Let us temporally introduce the notations $S_{\Psi}[\phi, \bar{g}]=S[\phi, \bar{g}]$ and $Z_{k \Psi}=Z_{k}$ to stress essential dependence of $S[\phi, \bar{g}]$ and $Z_{k}$ on gauge fixing procedure. In the functional integral

$$
Z_{k \Psi+\delta \Psi}=\int D \phi \exp \left\{\frac{i}{\hbar}\left(S_{\Psi}[\phi, \bar{g}]+\delta \Psi[\phi, \bar{g}] \hat{R}[\phi, \bar{g}]+J \phi+\Sigma \mathcal{L}_{k}(\phi, \bar{g})\right)\right\},
$$

we make use the change of integration variables in the form of BRST transformations but trading the constant parameter $\Lambda$ by functional

$$
\Lambda[\phi, \bar{g}]=\frac{i}{\hbar} \delta \Psi[\phi, \bar{g}] .
$$

Taking into account the corresponding Jacobian

$$
J[\phi, \bar{g}]=\exp \left\{-\frac{i}{\hbar} \delta \Psi[\phi, \bar{g}] \hat{R}[\phi, \bar{g}]\right\},
$$


omitting the subscript $\Psi$ we obtain the following equation

$$
\delta Z_{k}=\frac{i}{\hbar}\left(J_{i}+\Sigma \mathcal{L}_{k, i}(\widehat{q}, \bar{g})\right) R^{i}(\widehat{q}, \bar{g}) \delta \Psi[\widehat{q}, \bar{g}] Z_{k}
$$

describing the gauge dependence of the functional $Z_{k}=Z_{k}[J, \bar{g}, \Sigma]$. In (3.11) the notations

$$
\mathcal{L}_{k, i}(\phi, \bar{g})=\frac{\partial \mathcal{L}_{k}(\phi, \bar{g})}{\partial \phi^{i}}, \quad \widehat{q}^{i}=-i \hbar \frac{\delta}{\delta J_{i}}
$$

are used. From (3.11) it follows the important statement that the gauge dependence of $Z_{k}[J, \bar{g}, \Sigma]$ disappears when external sources are switched off, $J_{i}=\Sigma_{1}=\Sigma_{2}=0$.

In terms of the generating functional of connected Green functions, $W_{k}=W_{k}[J, \bar{g}, \Sigma]=$ $-i \hbar \ln Z_{k}[J, \bar{g}, \Sigma]$, the relation (3.11) takes the form

$$
\delta W_{k}=\left(J_{i}+\Sigma \mathcal{L}_{k, i}\left(\widehat{Q}_{k}, \bar{g}\right)\right) R^{i}\left(\widehat{Q}_{k}, \bar{g}\right) \delta \Psi\left[\widehat{Q}_{k}, \bar{g}\right] \cdot 1,
$$

where

$$
\widehat{Q}_{k}^{i}=\widehat{q}^{i}+\frac{\delta W_{k}}{\delta J_{i}}
$$

The modified effective average action, $\Gamma_{k}=\Gamma_{k}\left[\Phi_{k}, \bar{g}, F_{k}\right]$, is introduced through the double Legendre transform of $W_{k}$

$$
\begin{aligned}
& \Gamma_{k}\left[\Phi_{k}, \bar{g}, F_{k}\right]=W_{k}[J, \bar{g}, \Sigma]-J_{i} \Phi_{k}^{i}-\Sigma_{\ell}\left(\mathcal{L}_{k}^{(\ell)}\left(\Phi_{k}, \bar{g}\right)+\hbar F_{k}^{\ell}\right), \\
& \Phi_{k}^{i}=\frac{\delta W_{k}}{\delta J_{i}}, \quad \hbar F_{k}^{\ell}=\frac{\delta W_{k}}{\delta \Sigma_{\ell}}-\mathcal{L}_{k}^{(\ell)}\left(\frac{\delta W_{k}}{\delta J}, \bar{g}\right), \quad \ell=1,2 .
\end{aligned}
$$

From (3.15), (3.16) it follows

$$
\frac{\delta \Gamma_{k}}{\delta \Phi_{k}^{i}}=-J_{i}-\Sigma_{\ell} \mathcal{L}_{k, i}^{(\ell)}\left(\Phi_{k}, \bar{g}\right), \quad \frac{\delta \Gamma_{k}}{\delta F_{k}^{\ell}}=-\hbar \Sigma_{\ell}
$$

The modified effective average action satisfies the following functional integro-differential equation

$$
\exp \left\{\frac{i}{\hbar}\left(\Gamma_{k}-\frac{\delta \Gamma_{k}}{\delta F_{k}} F_{k}\right)\right\}=\int D \phi \exp \left\{\frac{i}{\hbar}\left(S\left[\Phi_{k}+\phi, \bar{g}, F_{k}\right]-\frac{\delta \Gamma_{k}}{\delta \Phi_{k}} \phi-\frac{1}{2} \frac{\delta \Gamma_{k}}{\delta\left(\hbar F_{k}\right)} \mathcal{L}_{k}^{(2)}\left(\Phi_{k}, \bar{g}\right) \phi \phi\right)\right\}
$$

where

$$
\mathcal{L}_{k}^{(2)}\left(\Phi_{k}, \bar{g}\right)=\frac{\delta^{2} \mathcal{L}_{k}\left(\Phi_{k}, \bar{g}\right)}{\delta \Phi_{k} \delta \Phi_{k}}
$$

In what follows below the gauge dependence of $\Gamma_{k}(3.15)$ is analyzed from the point of solutions to the equation (3.18) which can be in principal found perturbatively in the form of loop expansions having in this case their own specific features due to the fact that appearing equations have the form of Clairaut-type equations (for detailed discussions see [35]). 
Let us introduce the full sets of fields $\mathcal{F}_{k}^{\mathcal{A}}$ and sources $\mathcal{J}_{\mathcal{A}}$ according to

$$
\mathcal{F}_{k}^{A}=\left(\Phi_{k}^{i}, \hbar F_{k}^{\ell}\right), \quad \mathcal{J}_{A}=\left(J_{i}, \hbar \Sigma_{\ell}\right) .
$$

From the condition of solvability of equations (3.17) with respect to the sources $J$ and $\Sigma$, it follows that

$$
\frac{\delta \mathcal{F}_{k}^{C}(\mathcal{J})}{\delta \mathcal{J}_{B}} \frac{\vec{\delta} \mathcal{J}_{A}\left(\mathcal{F}_{k}\right)}{\delta \mathcal{F}_{k}^{C}}=\delta_{A}^{B}
$$

One can express $\mathcal{J}_{A}$ as a function of the fields in the form

$$
\mathcal{J}_{A}=\left(-\frac{\delta \Gamma_{k}}{\delta \Phi_{k}^{i}}-\frac{\delta \Gamma_{k}}{\delta F_{k}^{\ell}} \frac{\delta L_{k}^{\ell}\left(\Phi_{k}, \bar{g}\right)}{\delta \Phi_{k}^{i}},-\frac{\delta \Gamma_{k}}{\delta F_{k}^{\ell}}\right)
$$

and, therefore,

$$
\left(G_{k}^{\prime \prime}-1\right)^{A C}\left(G_{k}^{\prime \prime}\right)_{C B}=\delta_{B}^{A}, \quad \frac{\vec{\delta} \mathcal{J}_{B}\left(\mathcal{F}_{k}\right)}{\delta \mathcal{F}_{k}^{A}}=-\left(G_{k}^{\prime \prime}\right)_{A B}, \quad \frac{\delta \mathcal{F}_{k}^{B}(\mathcal{J})}{\delta \mathcal{J}_{A}}=-\left(G_{k}^{\prime \prime}-1\right)^{A B} .
$$

Taking into account that due to the Legendre transform

$$
\delta W_{k}=\delta \Gamma_{k},
$$

the equation (3.13) in terms of modified effective average action, $\Gamma_{k}=\Gamma_{k}\left[\Phi_{k}, \bar{g}, F_{k}\right]$, rewrites as

$$
\delta \Gamma_{k}=-\left(\frac{\delta \Gamma_{k}}{\delta \Phi_{k}^{i}}+\frac{1}{\hbar} \frac{\delta \Gamma_{k}}{\delta F_{k}}\left(\mathcal{L}_{k, i}\left(\Phi_{k}, \bar{g}\right)-\mathcal{L}_{k, i}\left(\widehat{\Phi}_{k}, \bar{g}\right)\right)\right) R^{i}\left(\widehat{\Phi}_{k}, \bar{g}\right) \delta \Psi\left[\widehat{\Phi}_{k}, \bar{g}\right] \cdot 1,
$$

where

$$
\widehat{\Phi}_{k}^{i}=\Phi_{k}^{i}+i \hbar\left(G_{k}^{\prime \prime}-1\right)^{i B} \frac{\vec{\delta}}{\delta \mathcal{F}_{k}^{B}}
$$

From (3.26) it follows

$$
\left.\delta \Gamma_{k}\right|_{\frac{\delta \Gamma_{k}}{\delta \mathcal{F}_{k}}=0}=0
$$

the gauge independence of modified effective average action calculated on the its extremals. This very important property of $\Gamma_{k}$ is found within the standard perturbation theory accepted in Quantum Field Theory for evaluation of functional integrals. In its turn the FRG is considered as non-perturbative approach to quantum field theories when the effective average action should be found as a solution to the flow equation. Quite recently [5] it was proved that the effective average action depends on gauge at every value of the IR parameter $k$ including the fixed points making impossible physical interpretation of any results obtained in the standard formulation of the FRG for gauge theories. One meets with the same drawback considering the reformulation of the method based on the 2PI effective action when regulators are considered as sources to composite fields [7, 8]. In the next section we are going to introduce an alternative flow equation and to study its $k$-dependence. 


\section{Alternative flow equation and $k$-dependence}

The flow equation in the FRG is the basic relation describing the dependence of the effective average action on the IR parameter $k$. Let us derive an alternative flow equation for the $2 \mathrm{PI}$ effective action (3.15). To do this we start with differentiating the functional $Z_{k}=Z_{k}[J, \bar{g}, \Sigma]$ (3.4) with respect to $k$ and taking into account that only quantities $\mathcal{L}_{k}(\widehat{q}, \bar{g})$ through the regulators $R_{k}(\bar{g})$ depend on $k$, we obtain the flow equation for the functional $Z_{k}$

$$
\partial_{k} Z_{k}=\frac{i}{\hbar} \Sigma \partial_{k} \mathcal{L}_{k}(\widehat{q}, \bar{g}) Z_{k}
$$

In terms of the generating functional of connected Green functions $W_{k}=W_{k}[J, \bar{g}, \Sigma]=-i \hbar \ln Z_{k}$, the relation (4.1) rewrites in the form

$$
\partial_{k} W_{k}=\Sigma \partial_{k} \mathcal{L}_{k}\left(\widehat{Q}_{k}, \bar{g}\right) \cdot 1
$$

In terms of the modified effective average action, $\Gamma_{k}=\Gamma_{k}\left[\Phi_{k}, \bar{g}, F_{k}\right]$ the relation (4.2) takes the form

$$
\partial_{k} \Gamma_{k}=-\frac{\delta \Gamma_{k}}{\hbar \delta F_{k}} \partial_{k} \mathcal{L}_{k}\left(\widehat{\Phi}_{k}, \bar{g}\right) \cdot 1
$$

From (4.3) it follows

$$
\left.\partial_{k} \Gamma_{k}\right|_{\frac{\delta \Gamma_{k}}{\delta F_{k}}=0}=0
$$

The modified flow equation obeys the independence on the IR parameter $k$ when it is considered on extremals,

$$
\frac{\delta \Gamma_{k}}{\delta F_{k}^{\ell}}=0, \quad \ell=1,2 .
$$

This fact gives a hope that calculations with the modified effective average action at the fixed points may have a physical meaning sense in contrast with the standard FRG.

In the next section we will study the gauge dependence problem for the alternative flow equation.

\section{Gauge dependence of alternative flow equation}

Taking into account that functions $\mathcal{L}_{k}^{(1)}(h, \bar{g})$ and $\mathcal{L}_{k}^{(2)}(C, \bar{C}, \bar{g})$ do not depend on gauges, from (4.1) it follows that the gauge dependence of alternative flow equation for the functional $Z_{k}$ is described by the equation

$$
\delta\left(\partial_{k} Z_{k}\right)=\frac{i}{\hbar} \Sigma \partial_{k} \mathcal{L}_{k}(\widehat{q}, \bar{g}) \delta Z_{k}
$$


or using (3.11) as

$$
\delta\left(\partial_{k} Z_{k}\right)=\left(\frac{i}{\hbar}\right)^{2} \Sigma \partial_{k} \mathcal{L}_{k}(\widehat{q}, \bar{g})\left(J_{i}+\Sigma \mathcal{L}_{k, i}(\widehat{q}, \bar{g})\right) R^{i}(\widehat{q}, \bar{g}) \delta \Psi[\widehat{q}, \bar{g}] Z_{k}
$$

We find an inspected fact that the gauge dependence of flow equation disappears already when external sources to composite fields are switched off, $\Sigma_{1}=\Sigma_{2}=0$. The alternative flow equation for the functional $W_{k}$ reads

$$
\delta\left(\partial_{k} W_{k}\right)=\frac{i}{\hbar}\left(\Sigma \partial_{k} \mathcal{L}_{k}\left(\widehat{Q}_{k}, \bar{g}\right)-\partial_{k} W_{k}\right) \delta W_{k}
$$

or in the form

$$
\delta\left(\partial_{k} W_{k}\right)=\frac{i}{\hbar} \Sigma\left(\partial_{k} \mathcal{L}_{k}\left(\widehat{Q}_{k}, \bar{g}\right)-\partial_{k} \mathcal{L}_{k}\left(\widehat{Q}_{k}, \bar{g}\right) \cdot 1\right)\left(J_{i}+\Sigma \mathcal{L}_{k, i}\left(\widehat{Q}_{k}, \bar{g}\right)\right) R^{i}\left(\widehat{Q}_{k}, \bar{g}\right) \delta \Psi\left[\widehat{Q}_{k}, \bar{g}\right] \cdot 1 .
$$

In terms of the modified effective average action the gauge dependence of alternative flow equations can be presented as

$$
\delta\left(\partial_{k} \Gamma_{k}\right)=\frac{i}{\hbar}\left(-\frac{\delta \Gamma_{k}}{\hbar \delta F_{k}} \partial_{k} \mathcal{L}_{k}\left(\widehat{\Phi}_{k}, \bar{g}\right)-\partial_{k} \Gamma_{k}\right) \delta \Gamma_{k},
$$

or as

$$
\begin{aligned}
& \delta\left(\partial_{k} \Gamma_{k}\right)=-\frac{i}{\hbar^{2}} \frac{\delta \Gamma_{k}}{\delta F_{k}}\left(\partial_{k} \mathcal{L}_{k}\left(\widehat{\Phi}_{k}, \bar{g}\right)-\partial_{k} \mathcal{L}_{k}\left(\widehat{\Phi}_{k}, \bar{g}\right) \cdot 1\right) \times \\
& \times\left(\frac{\delta \Gamma_{k}}{\delta \Phi_{k}^{i}}+\frac{1}{\hbar} \frac{\delta \Gamma_{k}}{\delta F_{k}}\left(\mathcal{L}_{k, i}\left(\Phi_{k}, \bar{g}\right)-\mathcal{L}_{k, i}\left(\widehat{\Phi}_{k}, \bar{g}\right)\right)\right) R^{i}\left(\widehat{\Phi}_{k}, \bar{g}\right) \delta \Psi\left[\widehat{\Phi}_{k}, \bar{g}\right] \cdot 1
\end{aligned}
$$

From (5.5), (5.6) it follows

$$
\left.\delta\left(\partial_{k} \Gamma_{k}\right)\right|_{\frac{\delta \Gamma_{k}}{\delta F_{k}}=0}=0 .
$$

Therefore, the flow equation is gauge independent on extremals,

$$
\frac{\delta \Gamma_{k}}{\delta F_{k}^{\ell}}=0,
$$

and as well as additionally obeys the independence on the IR parameter $k$. These facts give a possibility for consistent application of the proposed quantization procedure in gauge theories to obtain physical meaning results. It is interesting to note that the gauge independence of $\Gamma_{k}$ found as a solution to the flow equation is expected with use only a part of the equations of motion (5.8). In its turn the independence of the flow equation on the IR parameter $k$ at the extremals (5.8) can be considered as a signal about $k$-independence of $\Gamma_{k}$ at the fixed points.

\section{Summary}

We have studied basic properties of the flow equation in gravity theories within reformulation of the standard FRG [1, 2] when following ideas of [4] the 2PI effective action with composite 
fields being densities of the regulator action is introduced. In contrast with the standard FRG [1, 2] and alternative approaches [7, 8] the proposed reformulation leads to the 2PI effective average action which possesses standard properties of gauge dependence in the perturbation theory and satisfies the alternative flow equation being $k$-and gauge independent at extremals. Speaking about possible reformulations of the standard FRG it is necessary to notice the papers [36, 37] where the quantization procedure is based on gauge invariant regularization of an initial classical action which guarantees the BRST symmetry and the gauge independence of $S$-matrix elements. Unfortunately absence of explicit procedure to arrive the gauge invariance of regularized initial action does not allow to consider this approach as a consistent quantization method [38]. Therefore at the moment we state that the proposed reformulation of the standard FRG should be considered as non-perturbative quantization of gauge theories successfully passing through the gauge dependence tests.

\section{Acknowledgments}

The work is supported by the Ministry of Education of the Russian Federation, project FEWF2020-0003.

\section{References}

[1] C. Wetterich, Average action and the renormalization group equation, Nucl. Phys. B352 (1991) 529.

[2] C. Wetterich, Exact evolution equation for the effective potential, Phys. Lett. B301 (1993) 90 .

[3] N. Dupuis, L. Canet, A. Eichhorn, W. Metzner, J.M. Pawlowski, M. Tissier, N. Wschebor. The nonperturbative functional renormalization group and its applications, arXiv:2006.04853 [cond-mat.stat-mech].

[4] P.M. Lavrov, I.L. Shapiro, On the Functional Renormalization Group approach for YangMills fields, JHEP 1306 (2013) 086.

[5] P.M. Lavrov, BRST, Ward identities, gauge dependence and FRG, arXiv:2002.05997 [hepth].

[6] P.M. Lavrov, Gauge dependence of effective average action, Phys. Atom. Nucl. 83 (2020) 1011.

[7] E. Alexander, P. Millington, J. Nursey, P.M. Safin, An alternative flow equation for the functional renormalization group, Phys. Rev. D100 (2019) 101702.

[8] P.M. Lavrov, Gauge dependence of alternative flow equation for the functional renormalization group, Nucl. Phys. B957 (2020) 115107.

[9] J.M. Cornwell, R. Jackiw, E. Tomboulis, Effective action for composite operators, Phys. Rev. D10 (1974) 2428. 
[10] P.M. Lavrov, S.D. Odintsov, The gauge dependence of the effective action of composite fields in general gauge theories, Sov. J. Nucl. Phys. 50 (1989) 332 (Yad. Fiz. 50 (1989) $536)$.

[11] P.M. Lavrov, Effective action for composite fields in gauge theories, Theor. Math. Phys. 82 (1990) 282 (Teor. Mat. Fiz. 82 (1990) 402).

[12] P.M. Lavrov, S.D. Odintsov, A.A. Reshetnyak, Effective action of composite fields for general gauge theories in BLT covariant formalism, J. Math. Phys. 38 (1997) 3466.

[13] M. Reuter, Nonperturbative evolution equation for Quantum Gravity, Phys. Rev. D57 (1998) 971.

[14] B.S. DeWitt, Dynamical theory of groups and fields, (Gordon and Breach, 1965).

[15] V.F. Barra, P.M. Lavrov, E.A. dos Reis, T. de Paula Netto, I.L. Shapiro, Functional renormalization group approach and gauge dependence in gravity theories, Phys. Rev. D101 (2020) 065001.

[16] L.D. Faddeev, V.N. Popov, Feynman diagrams for the Yang-Mills field, Phys. Lett. B25 (1967) 29.

[17] A.O. Barvinsky, D. Blas, M. Herrero-Valea, S.M. Sibiryakov, C.F. Steinwachs, Renormalization of gauge theories in the background-field approach, JHEP 1807 (2018) 035.

[18] C. Becchi, A. Rouet, R. Stora, The abelian Higgs Kibble Model, unitarity of the S-operator, Phys. Lett. B52 (1974) 344.

[19] I.V. Tyutin, Gauge invariance in field theory and statistical physics in operator formalism, Lebedev Inst. preprint N 39 (1975).

[20] R. Delbourgo, M. Ramon-Medrano, Supergauge theories and dimensional regularization, Nucl. Phys. 110 (1976) 467.

[21] K.S. Stelle, Renormalization of higher derivative quantum gravity, Phys. Rev. D16 (1977) 953.

[22] P.K. Townsend, P. van Nieuwenhuizen, BRS gauge and ghost field supersymmetry in gravity and supergravity, Nucl. Phys. B120 (1977) 301.

[23] P.M. Lavrov, A.A. Reshetnyak, One loop effective action for Einstein gravity in special background gauge, Phys. Lett. B351 (1995) 105.

[24] R. Jackiw, Functional evaluation of the effective potential, Phys. Rev. D9 (1974) 1686.

[25] N.K. Nielsen, On the gauge dependence of spontaneous symmetry breaking in gauge theories, Nucl. Phys. B101 (1975) 173.

[26] P.M. Lavrov, I.V. Tyutin, On structure of renormalization in gauge theories, Sov. J. Nucl. Phys. 34 (1981) 156 (Yad. Fiz. 34 (1981) 277).

[27] P.M. Lavrov, I.V. Tyutin, On generating functional Of vertex functions in the Yang-Mills theories, Sov. J. Nucl. Phys. 34 (1981) 474 (Yad. Fiz. 34 (1981) 850). 
[28] B.L. Voronov, P.M. Lavrov, I.V. Tyutin, Canonical transformations and the gauge dependence in general gauge theories, Sov. J. Nucl. Phys. 36 (1982) 292 (Yad. Fiz. 36 (1982) 498).

[29] R.E. Kallosh, I.V. Tyutin, The equivalence theorem and gauge invariance in renormalizable theories, Sov. J. Nucl. Phys. 17 (1973) 98 (Yad. Fiz. 17 (1973) 190).

[30] B.S. De Witt, Quantum theory of gravity. II. The manifestly covariant theory, Phys. Rev. 162 (1967) 1195.

[31] I.Ya. Arefeva, L.D. Faddeev, A.A. Slavnov, Generating functional for the s matrix in gauge theories, Theor. Math. Phys. 21 (1975) 1165 (Teor. Mat. Fiz. 21 (1974) 311).

[32] L.F. Abbott, The background field method beyond one loop, Nucl. Phys. B185 (1981) 189.

[33] P.M. Lavrov, I.L. Shapiro, Gauge invariant renormalizability of quantum gravity, Phys. Rev. D100 (2019) 026018.

[34] B.L. Guacchini, P.M. Lavrov, I.L. Shapiro, Background field method for nonlinear gauges, Phys. Lett. B797 (2019) 134882.

[35] P.M. Lavrov, B.S. Merzlikin, Legendre transformations and Clairaut-type equations, Phys. Lett. B756 (2016) 188.

[36] T.R. Morris, Quantum gravity, renormalizability and diffeomorphism invariance, SciPost Phys. 5 (2018) 040.

[37] Y. Igarashi, K. Itoh, T.R. Morris, BRST in the exact renormalization group, Prog. Theor. Exp. Phys. (2019).

[38] P.M. Lavrov, RG and BV-formalism, Phys. Lett. B803 (2020) 135314. 